\title{
La función pública directiva ante el modelo emergente de "federalismo flexiblen en Europa
}

Eduardo Zapico Goñi *

\section{Introducción: la complejidad en las} negociaciones europeas como un reto para la función pública directiva

En otro artículo de este número de GAPP el Prof. Metcalfe propone la emergencia de un modelo de Europa que califica de "Federalismo flexible" que está lejos de imitar los modelos nacionales de tipo federal. Modelos tradicionales que exigen la transferencia de poder y competencias ejecutivas a las instituciones federales centrales. El modelo federal de tipo flexible no exigiría aumentos considerables de recursos humanos y financieros en las instituciones centrales de esa federación. Su funcionamiento no depende de un poder jerárquico europeo legitimado en la norma.

La propuesta parte de la hipótesis de que los Estados miembros van a retener una identidad y autonomía propia en ciertas áreas y van a compartir responsabilidades a través de la cooperación y la gestión conjunta de ciertas políticas comunitarias en otras áreas. La eficacia del sistema va a depender de un mayor compromiso con los valores colectivos europeos por parte de los Estados miembros y de sus Funciones públicas. El nuevo modelo federal está exigiendo una mayor participación de los Estados miembros en la defensa de intereses pluralistas tanto en la fase de formulación como de implantación de las políticas europeas. De hecho, se está percibiendo el nacimiento y creci- miento de redes o comunidades de políicas europeas que trascienden transversalmente los límites tradicionales de la nación Estado. Se exige una gestión por redes más que por jerarquías para alcanzar la eficacia en el funcionamiento de las instituciones europeas.

Cada vez en mayor medida la Unión Europea depende de unas relaciones pluralistas intergubernamentales tanto a nivel de formulación de políticas comunitarias como a nivel de puesta en marcha de esas políiticas. La Comisión depende de la cooperación de los Estados miembros en las tres fases del ciclo de toda política pública: formulación, ejecución y control. Una de las fuentes de complejidad más importantes con que se enfrentan los directivos públicos que defienden la eficacia colectiva en Europa es el reto de tener que diseñar políticas que tienen que ser implantadas de manera consistente o coherente en una serie de países con grandes diferencias en sus aparatos administrativos, tanto desde el punto de vista estructural como cultural e institucional.

La discusión hoy no debe radicar en si se necesita un federalismo que implique la construcción de un supraestado sino en las características institucionales y culturales de una nueva forma de federalismo que permita una dirección sin mando (guidance without command), combinando la autodirección y la dirección compartida, entre múltiples niveles de gobierno. Este proceso que MeTCAIFE llama de federalismo flexible se percibe tanto a nivel comunitario o europeo como a nivel subcentral o regional dentro de cada Estado miembro.

La adaptación de la Función Pública a un proceso de federalización flexiblen (Les MeTcAlfE, GAPP, 1996) de Europa está relacionado, entre otros factores, con lo que se viene denominando la globalización de la función pública. Se habla análogamente de la intemacionalización a nivel de la región europea para 
referirse a la necesidad de un nuevo estilo de trabajo y papel a jugar por la mayoría de los funcionarios directivos del Estado y Administraciones territoriales.

Las dificultades y obstáculos que encuentran los funcionarios directivos al participar en las negociaciones europeas es mayor de lo que suele pensarse. La formulación de las políticas comunes es el resultado de un proceso de múltiples interacciones y que podrían calificarse de complejas (Walton y MCKeARCY, 1965: 133). Esto implica la necesidad de tener en cuenta los siguientes factores de dificultad:

a. Naturaleza técnica y bumana. Se superponen componentes técnicos y humanos (valores, actitudes, sentimientos, percepción de penalizaciones y recompensas según el comportamiento de los participantes, etc.). Ambos componentes influyen de manera relevante en el resultado de las negociaciones y están o se presentan superpuestos. Asimismo, ambos influyen en la evolución del grado de confianza a lo largo de todo el proceso de negociación;

b. Proceso continuado. Los acuerdos se prolongan en el tiempo. Las negociaciones tienen una naturaleza continuada. Hay una conexión clara entre la fase de negociación previa al acuerdo y la fase posterior a cada decisión. Los acuerdos en ocasiones se revisan o se reinterpretan durante la fase de implementación. Por otro lado, la interacción entre los distintos Estados miembros europeos se plantea como una serie sucesiva e iterativa de negociaciones concatenadas en el tiempo, tanto desde el punto de vista del objeto como de los sujetos que la practican o no son fácilmente distinguibles;

c. Mezcla de intereses nacionales y europeos. La agenda de negociación tiene una doble naturaleza. En cada tema se pueden identificar aspectos conflictivos o tensiones entre intereses nacionales incompatibles y componentes colectivos en los que se representan intereses europeos. Resulta dificil identificar y compatibilizar los intereses nacionales con los intereses colectivos. La adaptación de una función pública al proceso decisorio europeo no es sólo una cuestión de preparación para competir con los demás Estados miembros sino también capacidad para cooperar en la identificación y construcción de los intereses globales. En ocasiones el planteamiento de la Administración de un Estado miembro responde a la consigna de "que no descubran nuestras debilidades", cuando de hecho la debilidad de la integración europea puede ser debida a las dificultades de comunicación y conocimiento entre las distintas Administraciones. Las posturas de competición racional en defensa del máximo beneficio nacional, independientemente del efecto que tenga en Eu- ropa, provoca que todos de manera colectiva salgan perdiendo.

d. Preparación de los intereses nacionales. Los actores que participan directamente en la negociación representan organizaciones (ya sean departamentos ministeriales, agencias descentralizadas, otros niveles de gobierno, intereses privados, etc.), cuyos miembros influyen en su comportamiento negociador y ejercen múltiples presiones a menudo incompatibles. En la medida en que las instancias nacionales no consigan integrar los intereses internos previamente a la defensa de una postura en las negociaciones europeas lo más probable es que en la fase de implantación se planteen dificultades para la puesta en práctica de las políticas comunes. Si la postura defendida por un delegado no representa o sintetiza los intereses de los niveles de Gobierno, grupos o agencias que posteriommente van a ser responsables de la ejecución de las decisiones europeas, lo más probable es que en la fase de implantación surjan tensiones y resistencias que debilitan el beneficio para el Estado miembro.

Las reuniones de negociación entre distintos funcionarios de los diversos Estados miembros que participan en el proceso de integración representan tan sólo una pequeña parte del proceso global de negociación. El encuentro con las contrapartes de otros países tan sólo representa la punta del iceberg del proceso de negociación. La preparación de las posturas que finalmente se defiendan en la mesa de negociación debería ir precedida de un enorme esfuerzo de recogida y análisis de información, procedente ya sea de grupos nacionales o correspondientes a otros paises. La definición de la postura o el interés nacional no es algo evidente en sí mismo ni se acepta de manera automática e indiscutible dentro de cada Estado miembro. La postura de cada país exige y depende de un estrecho contacto e interacción entre los centros de decisión y coordinación a nivel nacional. La eficacia de la estrategia de negociación de un Estado miembro depende de un esfuerzo prolongado e intenso de preparación interna que permita integrar diversos intereses intranacionales.

La dificultad de sintetizar los intereses nacionales se acentúa en el proyecto de negociación europea. En la medida que se profundice en el proceso de integración europea mayor será la confusión entre la política exterior y la política intema. Los Ministerios de Asuntos Exteriores y el Departamento de Comercio Exterior no son los únicos involucrados en el proceso de negociaciones. No parece realista centralizar toda la actuación preparatoria en uno de estos centros salvo riesgo de sobrecarga de trabajo y fomento de las resistencias burocráticas a un exceso de centralización. Aunque algunas decisiones puedan prepararse de manera independiente por algún departamento, en general la creciente interdependencia provocada por el proceso de internacionalización 
exigirá una mayor coordinación entre los distintos departamentos para participar con eficacia en las negociaciones europeas. La preparación interna de las posturas de negociación de cada Estado miembro requiere de por sí todo un proceso también de negociación a nivel intranacional.

El hecho de que se desarrollen con eficacia estas negociaciones internas tiene una doble proyección. No sólo se garantiza la coherencia global de los intereses nacionales sino que además se facilita el funcionamiento eficaz del proceso decisorio a nivel europeo. La debilidad de la capacidad de los funcionarios directivos de ejercer un rol integrador a nivel nacional puede provocar no sólo el perjuicio de los intereses nacionales sino además el perjuicio para el funcionamiento global de la política europea, tanto en la fase de formulación como en la de implementación. Existen estudios que muestran la correlación existente entre dificultades de coordinación interna en la fase ascendente y la debilidad posterior de la puesta en práctica de las decisiones comunitarias (SIEDENTOPH y ZULER, 1988: 5). En las siguientes páginas se analizan las peculiaridades de las negociaciones europeas con que se enfrentan los funcionarios directivos y los distintos estilos de decisión que mejor se adaptan a ellas.

\section{La integración europea como un problema de gestión de un proceso de negociaciones europeas}

Tradicionalmente la negociación diplomática se ha considerado como un proceso de interacción interpersonal en el que el objetivo es la manipulación, persuasión y convicción de la parte contraria. El truco y la habilidad para engañar sutilmente a la parte contraria ha sido la dimensión clásica más asociada con el trabajo del diplomático —aa maquinación diplomátican- La realidad en la que se desarrolla la negociación exterior tras un proceso de europeización o regionalización internacional es mucho más compleja: no es bilateral sino pluralista; se realiza bajo un seguimiento constante por parte de la Administración y los grupos de intereses nacionales y los temas sujetos a la negociación internacional van mucho más allá de los de representación, soberanía territorio, etc., incluyendo múltiples aspectos sectoriales y tecnológicos que escapan a los límites clásicos de la diplomacia (WINHAM, 1977).

Como hemos visto en la introducción, la preparación y gestión de las negociaciones europeas exige responder a una cre- ciente interdependencia y politización en la toma de decisión. Un síntoma claro de esta interdependencia es el hecho de que cada vez es mayor la consideración y tratamiento de problemas políticos y económicos a nivel europeo más que a nivel doméstico. Las decisiones europeas son una extensión de la formulación de políticas nacionales y/o viceversa. El voto por mayoría calificada aumenta la interdependencia en la toma de decisiones comunitarias. Asimismo, la toma de decisiones y negociación por paquetes de temas diversos hace muy necesario gestionar lo que podríamos llamar una interdependencia forzada o artificial. Como consecuencia de la difuminación de los límites entre política exterior y política intema, la negociación internacional se politiza. La postura nacional es difícil de reconciliar. Cada departamento o sector percibe de manera variada las presiones externas. Los representantes de cada Estado tienen que desarrollar una habilidad considerable para gerenciar múltiples conflictos internos, reconciliar una pluralidad de intereses y demandas de grupos de presión nacionales.

Por otro lado, exige la participación de toda la función pública. El número de funcionarios involucrados en la negociación internacional aumenta y va más allá de la mera representación diplomática. El proceso de negociación se despersonaliza. La eficacia de los negociadores deja de depender sólo de su capacidad de maquinación y pasa a depender en gran medida de la capacidad de gestión de múltiples procesos de negociación interrelacionados a distintos niveles de Gobierno y más allá de los límites al Sector Público.

Otro reto de gran importancia es el carácter desestructurado del contexto que enmarca las negociaciones europeas. Se podría describir la negociación diplomática tradicional mediante una línea continua en la que se representan dos posturas iniciales claras y opuestas al final de cada uno de los extremos de dicha línea y en el que algún punto intermedio representara el acuerdo o compromiso final. Las concesiones son cruciales y se dan mediante estrategias de convergencia que responden a expectativas sobre probables compromisos basados en percepciones de la reacción del adversario. La noción de negociación representada por una línea continua pasa a sustituirse por la imagen de un puzzle. La línea continua representa una situación muy estructurada. Sin embargo, las negociaciones europeas son más bien inciertas y desestructuradas. Las dimensiones que caracterizan un contexto desestructurado de negociación son, entre otras, la multiplicidad de actores con diversidad de intereses, la difusa delimitación de autoridad, la relativa inestabilidad e incertidumbre en cuanto a los efectos o utilidad de las posturas o propuestas de decisión, etc. (P. BERMan, 1980, p. 329). La naturaleza de los posibles resultados, por no mencionar los propios temas o asuntos a tratar, son difusos o desconocidos. En muchas situaciones los delegados negocian precisamente para descubrir cuáles son los temas a discutir. Los negociadores 
no disponen de una posición inicial clara y una estrategia consciente de concesiones por anticipado. El concepto de línea continua y convergencia sugiere la existencia de certidumbre. Sin embargo, el negociador europeo se mueve más bien por la vía de la prueba y el error. Se presta mayor atención al proceso de estructuración y guía de la propia negociación; se crean jerarquías de negociación (prioridades de negociación, programa temporal, etc.). La agenda de negociación tiene una doble naturaleza: conflictiva entre intereses nacionales y colaboradora en pro del interés colectivo.

\begin{tabular}{|l|l|l|}
\hline \multicolumn{2}{|c|}{ Complejidad del contexto que enmarca las negociaciones europeas } \\
\hline \multicolumn{1}{|c|}{ CARACTERISTICAS } & \multicolumn{1}{|c|}{ NEGOCIACIÓN DIPLOMATICA TRADICIONAL } & NEGOCIACIÓN EUROPEA \\
\hline IMAGEN & CONTINUUM & PUZZLE \\
SITUACIÓN & ESTRUCTURADA & DESESTRUCTURADA COMPLEJIDAD \\
ÁMBITO & BILATERAL & PLURAL \\
LIMITES POLITICA NACIONAL Y EXTERIOR & CLAROS & DIFUSOS \\
DURACIÓN & ALSLADA & CONTINUADA \\
OBJETIVO & CLARO & DIFUSO \\
ACTITUD & COMPETTTTVA & MIXTA \\
\hline
\end{tabular}

\begin{tabular}{|l|l|l|}
\hline \multicolumn{3}{|c|}{ Negociaciones europeas como un proceso de toma de decisiones } \\
para reducir la incertidumbre \\
\hline \multicolumn{1}{|c|}{ CARACTERISTICAS } & \multicolumn{1}{|c|}{ NEGOCIACIÓN DIPLOMÁTICA TRADICIONAL } & NEGOCIACIÓN INTEGRACIÓN EUROPEA \\
\hline ESTLLO & CONCESIONES Y COMPROMISO & BÚSQUEDA DE INFORMACIÓN PRUEBA Y \\
ERROR & $\begin{array}{l}\text { EXPLORATORIA COLABORADORA } \\
\text { SUJETOS }\end{array}$ & $\begin{array}{l}\text { TODOS LOS FUNCIONARIOS } \\
\text { CAPACIDADES }\end{array}$ \\
ALCANCE & $\begin{array}{l}\text { DIPLOMÁTICOS } \\
\text { PERSUASION }\end{array}$ & $\begin{array}{l}\text { GESTIÓN DEL PLURALISMO (MANAGEMENI) } \\
\text { RESULTADOS A LARGO PLAZO }\end{array}$ \\
\hline
\end{tabular}

La negociación se plantea ahora como un proceso necesario para reducir la incertidumbre creada por esta situación de creciente diversidad e interdependencia. El objetivo de la negociación pasa a ser la exploración y búsqueda de información (WINHAM, 1977). Gran parte del esfuerzo de negociación se dedica a la formulación de acuerdos que permitan estructurar el futuro. Claramente seguirán existiendo negociaciones para resolver disputas y conflictos concretos (cuotas de pesca, tarifas de importación, exportación fuera de Europa), pero en general estas negociaciones se realizarán en un contexto de reglas de decisión negociadas materializadas en forma de tratados o estilos de actuación consolidados.

Con la reducción de la incertidumbre se pretende aumentar la estabilidad de las relaciones europeas. Conforme aumente la interdependencia por el avance de la integración cada vez será mayor la presión para crear valores y estructuras a nivel europeo que sirvan para superar la incertidumbre de la acción unilateral.
Esto requiere la negociación de las propias reglas de negociación para alcanzar acuerdos sobre toma de decisiones colectivas a nivel europeo. La integración es un proceso de reestructuración de las actitudes de negociación (WaLTON y MCKERSIE, 1965) que anticipa el tratamiento de futuros conflictos o situaciones de bloqueo. La pérdida de autonomía en la política monetaria y de tipos de cambios a nivel nacional propuesta por los Estados Miembros en el Tratado de Maastricht puede ser interpretada como un intento de reducir la incertidumbre financiera. Los criterios de Maastricht para la convergencia y procedimientos de seguimiento de déficit excesivo suponen un marco de resolución de futuros conflictos comunitarios.

Tradicionalmente la negociación diplomática se desarrollaba con el fin de resolver situaciones de crisis. La diplomacia clásica implica la postura de una salida de negociación clara de las partes en litigio y la realización de movimientos de convergencia hacia una postura o posición de compromiso mediante con- 
cesiones realizadas paso a paso. El esfuerzo de cada negociador se centra en mantener al máximo su postura o posición inicial y simultáneamente conseguir un resultado que sea mutuamente aceptable. Este proceso de negociación clásico enfatiza las estrategias y tácticas de competición, concesión y la habilidad de persuasión. Este modelo sigue siendo parcialmente válido en la política internacional. Sin embargo, la negociación europea en una situación de complejidad exige reemplazar las estrategias y tácticas de persuasión por las de búsqueda de información, preocupación por el propio proceso de negociación y los resultados a largo plazo. Los negociadores no pueden calcular con precisión los resultados por que éstos son desconocidos, inciertos y distantes. El proceso de negociación supone en sí una búsqueda exploratoria de soluciones aceptables en la que la estrategia es más bien una cuestión de anticipar y evitar soluciones no atractivas más que el intentar cambiar la postura del adversario.

Otra implicación para la gestión de la negociación europea como un proceso de reducción de incertidumbre es el hecho de que se necesita desarrollar una percepción común y valores europeos más que el intercambio de concesiones en pro de un compromiso rápido entre los Estados miembros. Se trata de negociar sobre la •definición de la situación., implica compartir información y visión incluso antes del esfuerzo de elaborar la agenda de negociación. Las teorías sobre cómo construir una percepción común de problemas complejos son probablemente mucho más útiles que las teorías de enfrentamientos competitivos. Se necesita prestar mayor atención al desarrollo de reglas y actitudes de negociación. Esto implica el reconocimiento de la naturaleza continuada de las negociaciones europeas. Análogamente se tiene que desarrollar un lenguaje de negociación que permita facilitar la comunicación y las estructuras o modelos de conocimiento. Este lenguaje de aceptación colectiva son mecanismos de simplificación. La negociación europea es una fase más del proceso de formulación de políticas nacionales, y los negociadores se comportan más bien como funcionarios del Estado que como diplomáticos clásicos.

Las negociaciones europeas se desarrollan de manera análoga a un proceso de aprendizaje organizacional. Las partes negociadores representan burocracias gubernamentales que confrontan nuevos problemas a superar mediante una sucesión de encuentros que permiten elevar el grado de conocimiento y capacidad para su resolución: recogida y almacenamiento de información, desarrollo de procesos de comunicación, adaptación de objetivos a las posibilidades de cada situación, etc. Cada delegado aprende sobre la percepción de los problemas de los delegados de otros países, sus prioridades, valores, etc. Cada equipo de negociación desarrolla capacidad y conocimiento que facilitan la negociación. La naturaleza y práctica de la negociación europea supone un cambio de énfasis con la integración. El proceso se institucionaliza y es más parecido a una prolongación de la propia toma de decisiones a nivel nacional.

En la medida en que haya y se reconozca la interdependencia y diversidad de intereses la negociación y el conflicto en la integración europea es inevitable. la capacidad de gestión de conflictos y de dirección de procesos de negociación es un componente fundamental para la Unión Europea. En el apartado siguiente se analizan los posibles estilos de decisión para adaptarse a este nuevo contexto de la función pública directiva. Pero el problema no es la existencia de conflictos sino las disfunciones que éstos provocan (distorsionar la información, evitar la consulta más allá de la exigida reglamentariamente, reducir la interacción, etc...) por su no tratamiento o tratamiento inadecuado. En la medida en que el directivo público haga más transparente las posturas potencialmente complementarias y las interdependencias mayor será la propensión a la resolución conjunta de problemas (WaLTON, R. y DutTon, J., 1969, p. 73).

\section{Estilos de función directiva para la integración europea}

La cuestión que se trata de dilucidar aquí es la de cómo deben participar los funcionarios de una Administración en las negociaciones europeas con éxito. Es importante adelantar que este análisis es colectivo, referido al equipo directivo, e implica requisitos de comportamiento que se entienden como globales de la función pública de una Administración nacional o europea, no se plantea de manera individualizada para cada funcionario. El colectivo de gestores públicos en una Administración puede presentar un mapa desequilibrado o no ajustado al desafío de la integración. El marco conceptual que se presenta a continuación es útil para la identificación de estos desequilibrios y facilita vías para su solución.

Como ya se ha argumentado, el desafío de la gestión pública ante la europeización se asemeja al trabajo directivo en una situación pluralista definida no tanto por relaciones jerárquicas como por relaciones de interdependencia. Con la integración se está trabajando en un entorno pluralista que requiere la creación de redes de relaciones de trabajo, networking. La pregunta clave a plantearse es cómo crear estas redes, cómo activarlas y cómo orientar su trabajo de manera beneficiosa. O lo que es lo mismo, cómo se puede influir en la formulación y el desarrollo de la política comunitaria y cuál es la capacidad de respuesta del equipo de gestores públicos en un Estado miembro. La pregunta de partida sería qué posibles estilos y con qué limitacio- 
nes se pueden identificar en el gestor público al confrontar la integración europea (METCALFE, 1989).

a. El funcionario burócrata. mantiene una perspectiva formalista dando prioridad a la estabilidad en los procedimientos de trabajos tradicionales. No presta atención a los cambios en su entomo y a la necesidad de innovación. Su actitud es pasiva o reactiva, aplicando rutinas operativas sin crítica o análisis. Su objetivo es aplicar con equidad reglas y procedimientos. Su nivel o capacidad de adaptación a la integración europea es mínima.

b. El funcionario stecnócratan pretende la aplicación de la alternativa científica que maximice u optimice los resultados. Sus decisiones se basan en los méritos técnicos de cada opción en el marco de unas restricciones físicas o financieras. Su actitud es racionalista y se basa en la especialización o conocimiento experto. La eficacia de este estilo en el proceso de integración depende de los límites y restricciones políticas al no considerar otros factores que afectan a la gestión pública.

La mera optimización técnica es una aspiración no realista. El análisis y evaluación de políticas y programas públicos debería considerar también el examen de las condiciones de viabilidad de los programas o decisio- nes públicas. La optimización tradicionalmente se ha entendido de una manera muy restrictiva como para ser útil en la práctica. La decisión no significa acción. La decisión meramente técnica no tiene en cuenta futuros problemas de implementación y desprecia supuestamente la necesidad de apoyo político. Utiliza el análisis cuantitativo y aplica la lógica en la elección pero no considera factores políticos y organizacionales. Minimiza la responsabilidad por la consecución de resultados y/o implantación de decisiones.

c. El funcionario "político" tiene una perspectiva subjetiva. Analiza las preferencias y valores de los principales actores y/o afectados por cada decisión. Su actitud es incrementalista. No busca grandes variaciones ni decisiones alejadas o cualitativamente distintas de las preferencias reveladas al comienzo de cada negociación. Busca el compromiso dentro del campo limitado por las posturas iniciales de cada parte. Su objetivo es la negociación. Intenta identificar la decisión políticamente aceptable sin prestar atención al mérito técnico del resultado final. Su eficacia en un contexto internacionalizado depende de las posibilidades técnicas disponibles en cada política o programa público.

\begin{tabular}{|l|l|l|}
\hline \multicolumn{3}{|c|}{ Estilos de función directiva ante la integración * } \\
\hline \multirow{2}{*}{$\begin{array}{l}\text { CAPACIDAD } \\
\text { TECNICA }\end{array}$} & TECNÓCRATA & EMPRENDEDOR \\
\hline \multirow{2}{*}{ BUROCRÁTCO } & POLITICO \\
\hline \multicolumn{2}{|c|}{ HABHIDAD POIITICA } \\
\hline
\end{tabular}

- Les Metculfe, The An of the Feasible, EIPA, Maastncht, 1989 (documento interno).

\begin{tabular}{|c|c|c|c|c|}
\hline $\begin{array}{c}\text { ESTMOS FUNCION } \\
\text { DIRECTIVA }\end{array}$ & BURÓCRATA & TECNÓCRATA & POLÍTICo & EMPRENDEDOR \\
\hline VISION/DECISION & TRADICIONALISTA & CIENTIFFICO EXPERTO & $\begin{array}{l}\text {-SUBJETTVO } \\
\text { REPRESENTATIVO }\end{array}$ & $\begin{array}{l}\text { INNOVADOR } \\
\text { EXPLORATORIO }\end{array}$ \\
\hline $\begin{array}{l}\text { ACTTTUD/COMPOR- } \\
\text { TAMIENTO }\end{array}$ & $\begin{array}{l}\text { PASIVA } \\
\text { NO ANALITICA }\end{array}$ & $\begin{array}{l}\text { ESPECIALISTA } \\
\text { RACIONALISTA }\end{array}$ & DNCRENENTALISTA & $\begin{array}{l}\text { BÚSQUEDA ACTTVA } \\
\text { VIABLIDAD }\end{array}$ \\
\hline OBJETIVO & $\begin{array}{l}\text { CUMPLIMIENTO DEL } \\
\text { REGLAMENTO }\end{array}$ & $\begin{array}{l}\text { MAXIMIZACIONN } \\
\text { OPTTMIZACION }\end{array}$ & NEGOCIACIÓN & $\begin{array}{l}\text { INTEGRACIÓN } \\
\text { INTERMEDIACIÓN }\end{array}$ \\
\hline $\begin{array}{l}\text { ADAPTACIÓN A } \\
\text { EUROPEIZACIÓN }\end{array}$ & MINIMA & $\begin{array}{l}\text { DEPENDE DE } \\
\text { LIMTTACIONFS POLÍTICAS }\end{array}$ & $\begin{array}{l}\text { DEPENDE POSIBILIDAD } \\
\text { TÉCNICA }\end{array}$ & MÁXIMA \\
\hline
\end{tabular}

La opción políticamente preferible supone el análisis de aspiraciones de los actores más relevantes. Lo aceptable se presenta como más importante que lo óptimo. El mérito técnico se percibe como secundario. El regateo y la negociación más que como razonamiento técnico se utiliza como método de elección. Se buscan las so- 
luciones cuyo apoyo puede movilizarse. Se consideran las preferencias subjetivas, valores y percepciones de los principales actores.

d. El funcionario eemprendedor, ejerce un liderazgo pro-activo y exige una capacidad de innovación y búsqueda de soluciones viables. El objetivo no es la mera negociación rápida en busca de un compromiso que satisfaga de manera limitada las necesidades sino la intermediación y la gestión de interdependencia (networking) con la idea de mejorar la ganancia conjunta de las partes implicadas. Su capacidad para la adaptación a la integración es máxima. La función directiva tiene como prioritario el intentar que ambas condiciones las técnicas y políticas se cumplan. Exige la identificación y ensanche del solapamiento entre las soluciones políticamente populares y las técnicamente viables. Hay que satisfacer requisitos técnicos y políticos. Esto exige la identificación y análisis de oportunidades y desafíos que surgen en cada línea de negociación europea. Se necesita realizar un examen y valoración de la capacidad de respuesta actualmente disponible, en términos de recursos tales como el conocimiento experto. Parte de este examen consiste en la localización de las fuentes de procedencia de este conocimiento experto. Exige combinar simultáneamente una firme defensa de los intereses propios y la cooperación por satisfacer las necesidades de la parte contraria. Este estilo de función directiva exige trabajar conjuntamente con los representantes de otros gobiernos con el fin de intentar satisfacer plenamente los intereses de las partes enfrentadas y encontrar alternativas que cubran todas las pretensiones. Exige la exploración y la utilización de los puntos de desacuerdo con el fin de aprender respecto a las ideas e intereses de la parte contraria. Requiere un esfuerzo por identificar condiciones previas o requisitos que de no solventarse supondrían o fomentarian la competición. Esta modalidad de comportamiento directivo supone el reconocimiento de una situación de incertidumbre y que el conflicto de intereses es sólo -aparenten y su tratamiento puede proporcionar oportunidades de información para hallar soluciones imaginativas (K. Thomas, 1975: p. 899 y ss.).

Se requiere también el análisis de la utilidad que puedan proporcionar las posturas de negociación en términos de productos o servicios que son relevantes para sus receptores directos 0 indirectos (stake-bolders). Esto implica identificar quién sale beneficiado y cuál es el beneficio neto que se proporciona. El funcionario directivo emprendedor debe identificar los incentivos, recompensas o estímulos que motiven a posibles socios de coalición para cooperar en la misma línea de integración europea. Debe tratar de responder a la pregunta de qué contribu- ciones netas se pueden ofrecer a cambio de compromisos de apoyo.

Los mapas de las elecciones técnicamente deseables y el mapa de las elecciones políticas aceptables no son estáticos, cambian con el entomo. El cambio tecnológico puede traducirse en nuevas posibilidades. El apoyo político puede reducirse o ampliarse según la situación.

El trabajo del funcionario emprendedor. requiere también capacidad de liderazgo. La cuestión clave es decidir actuar por iniciativa propia o reaccionar a las iniciativas de otros. En un contexto de políticas públicas integrado a nivel europeo, no está claro quién juega el papel de liderazgo. En muchas ocasiones en la Comunidad Europea el liderazgo puede estar en manos de la Comisión, del Consejo, de algunos Estados miembros, del Parlamento europeo, de grupos de interés privado, etc. Incluso aunque no se desee jugar el papel de liderazgo, siempre será necesario conocer quién lo está realizando y con quién debe uno asociarse en coalición (MetCalfe, 1989). Esto implica un esfuerzo de:

a. Investigación/marketing, mediante: 1. La persuasión de socios potenciales sobre la validez del proyecto para que vayan modificando sus posturas adaptándolas a nuestro interés. 2. La identificación de las preferencias e intereses en la política discutida. 3. El desarrollo del propio tema y agenda en discusión mediante la interacción con otros socios potenciales y no sólo mediante el mero ajuste munuo (negociación bajo supuesto de suma positiva). 4. La identificación de resistencias potenciales 0 fuerzas de oposición.

b. Legitimación a través de la aceptación institucional y autorización formal política a nivel apropiado. El proceso de decisión en la CE es un laberinto que exige la identificación de los puntos críticos de decisión.

c. Intermediación o interconexión de las partes interesadas en la actuación o iniciativa europea. Esto implica la identificación de oferentes y demandantes de los recursos disponibles. No suele ser obvio dónde están ambos grupos, qué postura tienen y cómo pueden establecer contacto. Esta actuación del funcionario emprendedor va unida a la creación de equipos de trabajo con proyección europea.

d. Motivación o mantenimiento del compromiso para la aplicación de las decisiones y ajuste de la estrategia de implantación al contexto.

Es importante concluir recordando que la existencia de una comunidad profesional o de política europea no significa la existencia de una red de trabajo consolidada, estable y en funcionamiento (network). Con la integración no puede esperarse que 
automáticamente toda política europea genere una situación estructurada entre los miembros de la comunidad. Estas relaciones podrían ser totalmente difusas e inestables de tal manera que no permitieran observar las relaciones de dependencia existentes que condicionan la eficacia de la gestión pública. El estado estructurado de una red significa la existencia de:

a. Una preocupación común europea en la que se compartan intereses con una identidad propia colectiva.

b. Que se analicen la inclusión y exclusión de grupos.

c. Que se consideren miembros de la red a otros grupos de profesionales de otras políticas públicas (por ejemplo, una comunidad química e industrial o una comunidad agro-química) o incluso de distintas áreas o políticas (medio ambiente y agricultura).

d. La red o network puede variar en grado de integración según la estabilidad de las relaciones, su continuidad, las limitaciones de asociación, interdependencia interna, nivel de coordinación, etc.

\section{Conclusiones}

Cualquiera que sea la forma de integración europea, el proceso de integración requiere un nuevo estilo de función directiva en las Administraciones Públicas. Sin embargo, los programas de modemización administrativa y formación de funcionarios en los Estados miembros no han tenido muy en cuenta hasta ahora la europeización. Se han ignorado los nuevos retos que la integración europea conlleva para la gestión pública. En este trabajo se han presentado algunas dimensiones de las negociaciones europeas y estilo de adaptación de la función pública que se consideran fundamentales para desarrollar la capacidad de negociación y resolución de conflictos requerida para una buena participación en el proceso de toma de decisiones en Europa.

La realidad en la que se desarrolla la negociación internacional tras un proceso de europeización es mucho más compleja que la negociación diplomática tradicional. La negociación se plantea como un proceso necesario para reducir la incertidumbre creada por esta situación de creciente diversidad e interdependencia. El objetivo directo de la negociación pasa a ser la exploración y búsqueda de soluciones a problemas en contextos desestructurados. Gran parte del esfuerzo de negociación se dedica a la formulación de acuerdos que permitan estructurar el futuro con reglas de decisión negociadas, materializadas en for- ma de tratados o estilos de actuación de aceptación consolidada. Con la reducción de la incertidumbre se pretende aumentar la estabilidad de las relaciones europeas. Conforme aumente la interdependencia por el avance de la integración cada vez será mayor la presión para crear valores y estructuras a nivel europeo que sirvan para eliminar la posibilidad de acciones unilaterales. Esto requiere la negociación de las propias reglas de negociación para facilitar acuerdos colectivos. La integración europea es un proceso de reestructuración de las actividades de los funcionarios públicos que permita maximizar la capacidad de solución de problemas colectivos y reducir la incertidumbre sobre futuros conflictos o situaciones de bloqueo en Europa. El papel del funcionario directivo para dar respuesta a este desafío se considera fundamental.

Se han analizado los distintos estilos de dirección y su capacidad de adaptación a un entorno de integración europea negociada y conflictiva. El análisis del papel a jugar por lo que convencionalmente se ha denominado funcionario emprendedor, que combina las perspectivas y habilidades del funcionario "técnico" o racionalista, con las del funcionario "político", negociador, ofrece líneas de respuesta al tipo de capacidades requeridas en la función pública de los Estados miembros para maximizar su adaptación al proceso de integración europea. La respuesta ha sido analizada en torno al papel de un liderazgo de innovación e intermediación en la búsqueda activa de soluciones colectivas viables. La respuesta se ha elaborado en torno a la creación de redes de trabajo (networks), su activación y orientación de manera beneficiosa tanto a nivel nacional como europeo.

En definitiva, se ha tratado de dilucidar cómo participar con eficacia en las negociaciones europeas. El colectivo de gestores públicos en una Administración puede presentar un mapa desequilibrado o no ajustado al modelo emergente de integración europea. El marco conceptual que se ha presentado es útil para el diagnóstico y mejora de las capacidades de adaptación de las funciones públicas de los Estados miembros.

Este nuevo modelo de Gobierno exige un marco institucional original distinto que de alguna forma ya se va configurando tanto a nivel estructural como a nivel funcional. De la misma manera y afectando principalmente a las élites de gestores europeos (en Bruselas y en las distintas capitales de los Estados miembros) un nuevo estilo de decisión y comportamiento de los funcionarios en la formulación, implantación y control de las políticas públicas. El trabajo del funcionario directivo está cada vez más necesitado de capacidad creativa y de persuasión frente a su cometido tradicional de aplicación de la Ley o provisión eficiente de unos servicios públicos. 
- Oficina del Presupuesto de Presidencia del Gobiemo. Profesor Asociado Universidad Autónoma de Madrid.

\section{Bibliografia}

Bermav, P., Thinking about programmed and adaptive implementation. en $\mathrm{H}$. IN. GRAND y D. Mavi, Why Policies Succed of Fail?, London, Sage 1980, p. 329.

DROR, Yehezkel, Conducción del Estado bacia la integración, Integración Latinoamericana, INTAL, junio 1993.

JARA RoNCAT, Eduardo, La función diplomática, PNUD-CEPAL, Santiago, Chile, 1989.

KUKSBERG, Bemardo, Redesigning the State for Socioeconomic Development and Chan ge. A Strategic Agenda for Discussion, General Report of the second Intemational Conference on Administrative Sciences, Toluca, july 1993.

LAWRENCE Y LORSCH, Onganization and Environment, Irwin Inc., Illinois, 1969.

METCAIFE, Les, Gerencia de los procesos de integración: liderazgo gestion y coordina cion, Integración Latinoamericana, INTAL, junio 1993.

METCALF, Les, Trends in European Public Administration, EIPA, Maastricht 1993.
Metcalfe, Les, Tbe Ant of the Feasible, EIPA, Maastricht 1989.

Sundeutus, Bengt y Claes $W_{\text {LKIUND, }}$.The Nordic Community: the ugly duckling of regional cooperation, Joumal of Common Market Studies, Vol. XVIII, $\mathrm{n}^{\circ} 1$, september 1979.

Thomas, Kenneth, -Conflict and Conflict Management, en Marvi DunETTE (1975), Tbe Handbook of Industrial and Organizational Psycbology, Chicago, Rand McNally.

WALTON y DUTTON, The Management of Interdepartamental Conflict: a model and review Administrative Science Quarterly, Vol. 14, n. ${ }^{\circ}$ 1, march 1969.

WALTON y MCKERSIE, A Bebavioural Theory of Labor Negotiations, 1965.

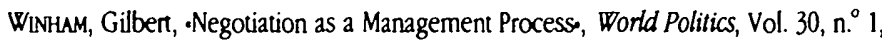
pp. 87 y ss., october 1997.

Working Document of the Commission, On a Strategie Programme on the Internal Market, Brussels, june 1993. 
Julho/Agosto 1997

Vol. 31 n.o 4 ISSN $0034-7612$

Periocidad bimestral

Revista da Escola Brasileira de Administraçao

Pública da Fundação Getulio Vargas

\section{EXPEDIENTE}

Director: Bianor Scelza Cavalcanti

Conselho Editorial: Anna Maria de Souza Monteiro Campos,

Aspásia Camargo, Bernardo Kilksberg. Heitor Chagas de

Oliveira, J. J. R. Fraústo da Silva, Marcos Cintra Cavalcanti de

Albuquerque, Paulo Roberto Motta, Sonia Maria Fleury

Teixeira, Tânia Fischer

Redatora: Deborah Moraes Zouain

EIDTORA FUNDAÇÃO GETULIO VARGAS

Editora executiva: Alzira Alves de Abreu

Coordenação editorial: Cristina Mary Paes de Cunha

Codidesque: Maria Izabel Penna Buarque de Almeida

Editoraçao electrônica: Jayr Ferreira Vaz e Simone Ranna

Revisao: Aleidis de Beltran e Fatima Caroni

Produçao gráfica: Helio Lourenço Netto

Gerente comercial: Carlos Hamilton Rocha

Assinaturas: Milton Gondim

Capa: Tira linhas studio

Índices nos quais a Revista de Administração Pública é citada: Bibliographie Latinoaméricaine d'Articles, $n .^{9} 22,1987$;

Handbook of Latin American Studies: Social Sciences, v. 41, 1979; Indices de Ciências Sociais/luperj, v. 9, 1989; International Bibliography of the Socials Sciences Economics; Sumários Correntes Brasileiros: Ciências Sociais e Humanas

CORRESPONDÊNCIA REDATORIAL

Inclusive originais de artigos (três vias):

REVISTA DE ADMINISTRAÇÃO PÚBLICA

Praia de Botafogo, 190 - sala 426-

CEP 22253-900

CORRESPONDÊNCIA COMERCIAL

FUNDAÇÃO GETULIO VARGAS/EDITORA

Caixa Postal 62.591 - CEP 22252-970

Rio de janeiro, RJ, DDG: (0800) 21-7777

e-mail: assine @ fgv.br

\section{FUNDAÇÃO GETULIO VARGAS \\ LIVRARIA J. CARNEIRO FELIPE}

Parida de Botafogo, 188

Rio de Janeiro, RJ - CEP 22253-900

Tel.: (021) 536-9105

LIVARARIA FARIA LIMA

Av. Nove de Julho, 2.029, Sao Paulo, SP

Tel.: (011) $281-7875$

Palavras do director

\section{ARTIGOS}

Os impactos das telecomunicações sobre as corporaçб̃es Industriais e financeiras

Carlos Alberto F. da Silva

Planejamento estratégico o planejamento participativo na gestão municipal: o caso do municíplo de Porto Alegre gestão 1989-93

Gilnei Luiz de Moura
Globo Rural -oportunidade para levantamentos de demandas de pesquisas e difusão de tecnologias: um estudo de caso

Adriana Maria de Aquino, Helvécio De-Polli e Renato Linhares de Assis

O modelo brasllelro de prestação de contas: por uma racionalização do processo

Flávio Lúcio Rodrigues da Silva

Estratégias para a implantaçăo e gerêncla de sistemas de informação de appio à tomada de decisoes

Osvaldo Maldonado Sanches

Gestão pública e cludadania: metodologias participativas em açå

Fernando Guilherme Tenório e Jacob Eduardo

Rozenberg

Gestão pública e cludadania: Iniciativas inovadoras na administração subnacional no Brasil

Marta Ferreira Santos Farah

Reflexoes sobre a Federaçao brasileira

Atilio Machado Peppe, Eduardo da Silva Pereira, Frederico

Raphael C. Durao Brito e Marcelo Ernandes Macedo

Fatores agilizadores e restritivos à atuaçăo da Justiça do Trabalho: um estudo exploratório

Cecilia Vescovi de Aragao

Novos modelos de gestao na prestação de serviços

públicos e o establecimiento do marco regulatório

Gloria Conforto

ESPAÇO INTERNACIONAL

Respensando el Estado para el desarrollo social: más allá de dogmas y convencionalismos

Bernardo Kilksberg

DOCUMENTO

O processo decisório de criaçao do Centro Cultural Banco do Brasil

Ana Heloísa da Costas Lemos

DEPOIMENTO

EBAP lança novo produto: Curso de Administraçăo

Avançada de Estados e Municipios

Istvan Karoly Kasznar

SEÇÕES ESPECIAIS

Programa de Estudos em Gestro Soclal

Projeto Ciudadania e Municipalidade

Fernando Guilherme Tenório

Programa de estudos e pesquisas em reforma do

Estado e governança

Reforma administrativa: uma visao critica

Sonia Felury

A conjuntura das escolhas públicas

Estabilidade de mando versus instabilidade constitucional Jorge Vianna Monteiro

Produção e desenvolvimento nos estados e municíplos

7 Elementos básicos fiscais na mensuração do PIB municipal, estadual ou nacional: approach simplificado

Istvan Karoly Kasznar

Os artigos assinados são de responsabilıdade exclusiva dos autores. E permitida a

23 reproduçào total ou parcial dos artigos desta revista, desde que citada a fonte. 